Working Paper 05-63

Economics Series 32

November 2005
Departamento de Economía

Universidad Carlos III de Madrid

Calle Madrid, 126

28903 Getafe (Spain)

Fax (34) 916249875

\title{
MISTAKES IN COOPERATION: THE STOCHASTIC STABILITY OF EDGEWORTH'S RECONTRACTING *
}

\author{
Roberto Serrano ${ }^{1}$ and Oscar Volij ${ }^{2}$
}

Abstract

In an exchange economy with a finite number of indivisible goods, we analyze a dynamic trading process of coalitional recontracting where agents maymake mistakes with small probability. We show first that the recurrent classes of the unperturbed (mistake-free) process consist of (i) all core allocations as absorbing states, and (ii) non-singleton classes of non-core allocations. Next, we introduce a perturbed process, where the resistance of each transition is a function of the number of agents that make mistakes -do not improve- in the transition and of the seriousness of each mistake. If preferences are always strict, we show that the unique stochastically stable state of the perturbed process is the Walrasian allocation. In economies with indifferences, non-core cycles are sometimes stochastically stable, while some core allocations are not. The robustness of these results is confirmed in a weak coalitional recontracting process.

Keywords:.. C7, D51, D61, D63

JEL classification:. stochastic stability, exchange economies, assignment problems, core, Walrasian equilibrium

* Acknowledgements: We thank Bob Aumann, Geoffroy de Clippel, Philippe Jehiel, Michihiro Kandori, Eric Maskin and Indra Ray for useful suggestions. Serrano acknowledges support from Fundaci'on Banco Herrero, Universidad Carlos III, NSF grant SES-0133113 and Deutsche Bank, and thanks Universidad Carlos III and CEMFI in Madrid and the Institute for Advanced Study in Princeton for their hospitality

\footnotetext{
${ }^{1}$ Brown University. Department of Economics, Providende, RI 02912, U.S.A. e-mail: roberto_serrano@brown.edu

${ }^{2}$ Iowa State University. Department of Economics e-mail: oscar@volij.co.il
} 


\title{
MISTAKES IN COOPERATION: the Stochastic Stability of Edgeworth's Recontracting*
}

\author{
Roberto Serrano ${ }^{\dagger}$ \\ Oscar Volij \\ This version: November 2005
}

\begin{abstract}
In an exchange economy with a finite number of indivisible goods, we analyze a dynamic trading process of coalitional recontracting where agents may make mistakes with small probability. We show first that the recurrent classes of the unperturbed (mistake-free) process consist of (i) all core allocations as absorbing states, and (ii) non-singleton classes of non-core allocations. Next, we introduce a perturbed process, where the resistance of each transition is a function of the number of agents that make mistakes - do not improvein the transition and of the seriousness of each mistake. If preferences are always strict, we show that the unique stochastically stable state of the perturbed process is the Walrasian allocation. In economies with indifferences, non-core cycles are sometimes stochastically stable, while some core allocations are not. The robustness of these results is confirmed in a weak coalitional recontracting process.
\end{abstract}

JEL classification numbers: C7, D51, D61, D63.

Keywords: stochastic stability, exchange economies, assignment problems, core, Walrasian equilibrium.

${ }^{*}$ We thank Bob Aumann, Geoffroy de Clippel, Philippe Jehiel, Michihiro Kandori, Eric Maskin and Indra Ray for useful suggestions. Serrano acknowledges support from Fundación Banco Herrero, Universidad Carlos III, NSF grant SES-0133113 and Deutsche Bank, and thanks Universidad Carlos III and CEMFI in Madrid and the Institute for Advanced Study in Princeton for their hospitality.

†Brown University, roberto_serrano@brown.edu, www.econ.brown.edu/faculty/serrano

${ }^{\ddagger}$ Iowa State University and Ben-Gurion University of the Negev, oscar@volij.co.il, www.volij.co.il 


\section{Introduction}

\subsection{Stochastic Stability in Economic Models}

The objective of this paper is to apply the concept of stochastic stability to non-strategic contexts where property rights play an important role in determining the outcome. Private ownership economies and cooperative games are two examples of the frameworks we have in mind. Stochastic stability has been introduced to game theory by Kandori, Mailath and Rob (1993), and Young (1993), who applied it to $2 \times 2$ games as a selection device. ${ }^{1}$ Since then it has been applied successfully to a wide variety of models (see Young (1998)). The concept of stochastic stability belongs to the "evolutionary" approach to explaining the emergence of equilibria, conventions or social norms. ${ }^{2}$ Within this approach, agents are not necessarily fully rational, but "programmed" to behave in a fixed way until they are replaced by other agents that, unless they are unlikely mutations, are better prepared to face their environment.

The usual application of stochastic stability to non-cooperative games fixes a one-shot game in normal form (strategy sets and payoff functions) played by myopic players. A stochastic process is specified to explain how players choose their strategies in each period. The process consists of three forces: inertia, selection and randomness. Inertia is the driving force that is in place most of the time: typically players simply repeat what they played in the last period, or copy the strategy played by his immediate ancestor playing the game, without really giving a thought to their choice. At times, however, the system is taken over by selection: a player may want to put some thought into his strategy, for example choosing it as a best response to a representative sample of past history. Finally, randomness appears as instances of mutations or experimentation in players' choices. The question posed then is what strategies are most likely to be played in the long run when this stochastic process is in place. These strategies are said to be stochastically stable, and have been shown to have a close relationship with interesting properties such as risk dominance.

Similarly, in this paper we consider a one-shot exchange economy (preferences and en-

\footnotetext{
${ }^{1}$ This methodology, based on the techniques developed for stochastic dynamical systems by Freidlin and Wentzell (1984), was first applied to evolutionary biology by Foster and Young (1990).

${ }^{2}$ Other references for evolutionary game theory in general are Weibull (1995), Vega Redondo (1996), Samuelson (1997) and Young (1998).
} 
dowments) with myopic agents that is repeated over time. We are interested in the evolution of the allocations, when they follow a stochastic process that is composed of the same three forces. The main force, within which the other two are embedded, can be seen as a flow of inertia. In each period the agents take their fixed initial endowments and trade them for a given consumption bundle, repeating the pattern of trade of the previous period. These bundles constitute a status-quo allocation which is consumed every period, unless something disturbs the system. Embedded in this flow of inertia there is a process of selection. In each period a coalition is chosen with some small probability and it has the opportunity to find a reallocation of its resources that makes its members better off. If they find one such reallocation they carry it out, thus sending the economy to a new status-quo allocation, which will be repeated each period until the next disturbance takes place. Finally, there is randomness represented by a process of mistakes. ${ }^{3}$ When a coalition is selected to look for a profitable recontract, there is a small probability that some of its members agree to a reallocation that makes them worse off. This persistent randomness ensures that the system does not get stuck at any given state. Instead, it keeps transitting all the time from one state to the next. Stochastic stability then identifies those states (allocations, in this case) that are visited by the system a positive proportion of time in the very long run.

Fixing the preferences and endowment of the economy allows us to compare the stochastically stable allocations to the ones prescribed by classical solution concepts, such as the core or Walrasian equilibrium. Therefore, our goal is to identify those allocations that will be visited by Edgeworth's recontracting process a positive proportion of time in the long run if mistakes are small probability events that all agents make all the time. ${ }^{4}$ We shall offer two kinds of results. First, under some conditions, stochastic stability will provide a new foundation of the Walrasian logic. And second, and perhaps more fundamentally because this is likely to be the result to be expected in general, stochastic stability highlights that some solution concepts -like the core- might be missing an aspect of coalitional stability that stochastic dynamics may help capture.

\footnotetext{
${ }^{3}$ Indeed, papers in cooperative game theory have no mistakes. We shall depart from this noble tradition.

${ }^{4}$ In contexts closer to a social choice model, where only preferences and not the endowments are part of the primitives, Ben-Shoham, Serrano and Volij (2004) and Kandori, Serrano and Volij (2004) explore related models that use mistakes in decision making or random utility maximization.
} 


\subsection{Coalitional Recontracting}

The question of what outcomes will arise in decentralized trade amongst arbitrary groups of agents dates back to Edgeworth (1881). His answer was the set of "final settlements" and it is what today we refer to as the core, i.e., the set of allocations "which cannot be varied with advantage to all the contracting parties." Although Edgeworth's definition of his "final settlements" applies to a static exchange economy, he motivates them with a description of a recontracting process that is inherently dynamic, and which received elegant formalizations in the analysis of Feldman (1974) and Green (1974). ${ }^{5}$ These authors show that under certain assumptions on the economy, their recontracting process converges to a core allocation. In contrast to these papers, ours considers the possibility of mistakes in agents' decision-making within the context of dynamic coalitional exchange.

In Feldman (1974) and Green (1974), a dynamic random process was imposed on a coalitional game. Starting from an arbitrary feasible allocation, the process allows each coalition to meet with positive probability in every period. When a coalition meets, they can choose to stay at the original allocation or move to a new allocation, feasible for them, if they all improve as a result. When this happens, the complement coalition is sent back to their individual endowments (in Feldman (1974)) or to a Pareto efficient allocation of their resources (in Green (1974)). The adjustment of resources of the complement coalition ensures that the path followed in utility space by the process is not monotonic, and renders the convergence question interesting and non-trivial.

In the present paper we analyze a dynamic recontracting process similar to those of Feldman (1974) and Green (1974), but applied to the class of housing economies introduced by Shapley and Scarf (1974), which have a finite set of allocations, amenable to the use of finite Markov chains. One interesting feature of these economies is that they do not satisfy some of the Feldman-Green conditions (free disposal of utility for every coalition). Hence, in these economies those recontracting systems and ours fail to converge to core allocations. Indeed, in the specification of the dynamics, some recurrent classes arise which consist solely of non-core allocations. In view of this emergence of a large number of recurrent classes, one is led to consider refinement techniques. Adding mistakes and resorting to stochastic

\footnotetext{
${ }^{5}$ One other aspect of dynamics and the core is provided by its extensive-form non-cooperative implementation (e.g., Perry and Reny (1994), Dagan, Serrano and Volij (2000)).
} 
stability is a way to accomplish this.

To be more precise, when we analyze the recontracting process free of mistakes, each core allocation constitutes an absorbing state, and in addition, we find recurrent classes consisting of cycles of non-core allocations. Bringing mistakes to bear, our results turn out to depend on the class of economies one considers.

In economies where preferences are strict, we are able to get a remarkable refinement of the set of recurrent classes by perturbing the system with mistakes. Our first main result is that the unique stochastically stable state of the recontracting process with mistakes is the Walrasian allocation, provided that serious mistakes (those where agents end up worse off as a result) are sufficiently more costly than minor ones (those where the agent joins a coalition to end up indifferent to how he started). At the heart of this result is the property of "global dominance" of the Walrasian allocation for economies with no indifferences, uncovered in Roth and Postlewaite (1977): for every feasible allocation of the economy, there exists a coalition that can (weakly) improve upon the status quo with their components of the Walrasian allocation. This is of course quite special to this setting, and one should not expect to be able to extend this result to general economies, yet the sufficient conditions assumed are of interest and a new foundation of Walrasian equilibria is provided.

The conclusions of our analysis are quite different in the more general case of economies with indifferences, where we obtain non-Walrasian results. We provide a series of examples to illustrate that the predictions of stochastic stability will not coincide with any of the classical solution concepts. In particular, we regard Example 5 as the other main result of the paper: it shows that non-core (and hence, non-Walrasian) cycles are sometimes stochastically stable, whereas some core allocations are not. This is worth stressing for two reasons. First, in recontracting with mistakes, the economy may frequently visit coalitionally unstable cycles: despite their being ruled out by the definition of the core, they represent classes of states in which the economy spends a very significant amount of time. Second, entire regions of the core will not be reached but a zero proportion of time in the very long run. That is, dynamics allows one to go beyond the core definition and classify allocations within the core in terms of how easy it is for the economy to get there. This suggests that there are different levels of "decentralization" behind each core allocation: for instance, whereas some may arise as a result of a simple sequence of trades and are therefore easy to reach, others will require complicated transactions, and hence one should not expect to reach them so 
easily. Also, to the best of our knowledge, this appears to be one of the first examples in the evolutionary literature where a non-singleton recurrent class turns out to be stochastically stable.

As a robustness check of our results in the coalitional recontracting process, we also study a second process based on weak coalitional recontracting. We find that stochastic stability always selects a non-empty subset of Walrasian allocations when the unperturbed process contains only singleton recurrent classes (in particular, in economies with only strict preferences, the only Walrasian allocation is the unique recurrent class of this unperturbed process). If the mistake-free process allows also non-singleton recurrent classes, however, stochastic stability is compatible with the appearance of cycles containing non-Walrasian allocations.

\subsection{Plan of the Paper}

The paper is organized as follows. Section 2 presents the model and basic definitions. Section 3 introduces the unperturbed recontracting process. Its perturbed version with mistakes is found in Section 4. Section 5 contains our result when preferences are strict. Section 6 focuses on economies with non-singleton indifference sets, and goes through a series of examples. To check the robustness of our results, Section 7 analyzes an alternative model of weak recontracting and mistakes. Section 8 discusses the relation of our dynamic model with Edgeworth's description of the recontracting process. Section 9 concludes.

\section{A Housing Economy}

We shall pose our questions in the context of the housing model of Shapley and Scarf (1974), a simple exchange economy with only indivisible goods (see Roth, Sonmez and Umver (2004) for a recent successful application of the model).

Formally, a housing economy is a 4-tuple $\mathcal{E} \equiv\left\langle N, H,\left(\succeq_{i}, e_{i}\right)_{i \in N}\right\rangle$, where $N$ is a finite set of individuals, $H$ is a finite set of houses with $|H|=|N|$, and for each individual $i \in N$, $\succeq_{i}$ is a complete and transitive preference relation over $H$, with $\succ_{i}$ denoting its associated strict preference relation and $\sim_{i}$ its indifference relation. Finally, $\left(e_{i}\right)_{i \in N}$ is the profile of individual endowments. 
A coalition $S$ of agents is a non-empty subset of $N$. The complement coalition of $S, N \backslash S$, will be sometimes denoted by $-S$. A feasible allocation for coalition $S$ is a redistribution of the coalitional endowment $\left(e_{i}\right)_{i \in S}$ among the members of $S$. Denote the set of feasible allocations for coalition $S$ by $A_{S}$. We simply write $A$ for $A_{N}$. An allocation $x \in A$ is individually rational if there is no individual $i \in N$ for whom $e_{i} \succ_{i} x_{i}$. An allocation $x \in A$ is a core allocation if there is no coalition $S$ and no feasible allocation for $S, y \in A_{S}$, such that $y_{i} \succ_{i} x_{i}$ for all $i \in S$. An allocation $x \in A$ is a strong core allocation if there is no coalition $S$ and no feasible allocation for $S, y \in A_{S}$, such that $y_{i} \succeq_{i} x_{i}$ for all $i \in S$ and $y_{j} \succ_{j} x_{j}$ for some $j \in S$. An allocation $x \in A$ is a Walrasian allocation if there exists $p \in \mathbb{R}_{+}^{H} \backslash\{0\}$ such that for all $i \in N p_{x_{i}}=p_{e_{i}}$, and for all $i \in N$ and for all $h \in H, h \succ_{i} x_{i}$ implies $p_{h}>p_{e_{i}}$. It has been shown (see Shapley and Scarf (1974)) that an allocation $x$ is Walrasian if and only if it can be obtained as a result of trading cycles. That is, if and only if there exists a partition of the set of agents $\left\{S_{1}, S_{2}, \ldots, S_{m}\right\}$ such that for every $k=1,2, \ldots, m$, $x_{S_{k}} \in A_{S_{k}}$, and for every $j \in S_{k}, x_{j} \succeq_{j} x_{i}$ for every $i \in S_{k} \cup \ldots S_{m}$.

In words, the agents in $S_{1}$ redistribute their endowments and get their most preferred houses; the agents in $S_{2}$ redistribute their endowments and get their most preferred houses out of the endowments of $S_{2} \cup \ldots S_{m}$, etc.

In the following sections, we shall define a perturbed Markov process for any given housing economy. The states of the process are the allocations of the housing economy. In each period a coalition of agents is selected at random and the system moves from one state to another when the matched agents recontract. In the unperturbed Markov process $\mathcal{M}^{0}$ of Section 3, agents do not make mistakes in their coalitional meetings: they sign a contract if and only if there is a strictly beneficial coalitional recontracting opportunity. In the perturbed process $\mathcal{M}^{\epsilon}$ of Section 4, agents will make mistakes with a small probability, and sign a contract with a coalition even when they do not improve as a result. In Section 7 we study Markov trading processes in which coalitions can find a weak (rather than strict) coalitional recontracting move.

It is often the case that an unperturbed Markov process (and it will certainly be the case for $\left.\mathcal{M}^{0}\right)$ has many stationary distributions. On the other hand for all $\epsilon \in(0,1)$, the 
perturbed process $\mathcal{M}^{\epsilon}$ is ergodic, which implies that it has a unique stationary distribution, denoted by $\mu^{\epsilon}$. This stationary distribution, which is independent of the initial state, represents the proportion of time that the system will spend on each of its states in the long run. It also represents the long run probability that the process will be at each allocation. In order to define the stochastically stable states, we check the behavior of the stationary distribution $\mu^{\epsilon}$ as $\epsilon$ goes to 0 . It is known that $\lim _{\epsilon \rightarrow 0} \mu^{\epsilon}$ exists and further it is one of the stationary distributions of the unperturbed process $\mathcal{M}^{0}$. The stochastically stable states of the system $\mathcal{M}^{\epsilon}$ are defined to be those states that are assigned positive probability by this limit distribution. We are interested in identifying these allocations because they are the ones that are expected to be observed in the long run "most of the time."

\section{An Unperturbed Recontracting Process}

Consider the following unperturbed Markov process $\mathcal{M}^{0}$, adapted from Feldman (1974) and Green (1974). In each period $t$, if the system is at the allocation $x(t)$, all coalitions are chosen with arbitrary, but positive, probability. Suppose coalition $S$ is chosen.

(i) If there exists an $S$-allocation $y_{S} \in A_{S}$ such that $y_{i} \succ_{i} x_{i}(t)$ for all $i \in S$, the coalition moves with positive probability to one of such $y_{S}$ in that period. Then, the new state is either

$$
\begin{aligned}
& x(t+1)=\left(y_{S}, x_{-S}(t)\right) \text { if } x_{-S}(t) \in A_{-S}, \text { or } \\
& x(t+1)=\left(y_{S}, e_{-S}\right) \text { if } x_{-S}(t) \notin A_{-S} .
\end{aligned}
$$

(ii) Otherwise, $x(t+1)=x(t)$.

The interpretation of the process is one of coalitional recontracting. Following a status quo, a coalition can form and modify it if all members of the coalition improve as a result. When this happens, upon coalition $S$ forming, the complement coalition $N \backslash S$ continues to have the same houses as before if this is feasible for them. Otherwise, $N \backslash S$ breaks apart and each of the agents in it receives his individual endowment. ${ }^{6}$ If after coalition $S$ gets together, all its agents cannot find any strict improvement, the original status quo persists.

\footnotetext{
${ }^{6}$ Our treatment of the complement coalition constitutes a small difference with respect to the processes in Feldman (1974) and Green (1974). Our results are robust to different specifications. For instance, this
} 
It is clear that the absorbing states of this unperturbed process are precisely the core allocations of the economy. However, the absorbing states are not the only recurrent classes of $\mathcal{M}^{0}$, as shown by the following example.

Example 1 Let $N=\{1,2,3\}$ and denote by $\left(e_{1}, e_{2}, e_{3}\right)$ the individual endowment allocation. Let the agents' preferences be as follows:

$$
\begin{aligned}
& e_{3} \succ_{1} e_{2} \succ_{1} e_{1} ; \\
& e_{1} \succ_{2} e_{3} \succ_{2} e_{2} ; \\
& e_{2} \succ_{3} e_{1} \succ_{3} e_{3} .
\end{aligned}
$$

Consider the following three allocations: $x=\left(e_{1}, e_{3}, e_{2}\right), y=\left(e_{2}, e_{1}, e_{3}\right)$ and $z=$ $\left(e_{3}, e_{2}, e_{1}\right)$. These three allocations constitute a recurrent class: if the system is at $x$, the state changes only when coalition $\{1,2\}$ meets, yielding $y$. At $y$, the system can move only to $z$, when coalition $\{1,3\}$ meets. Finally, the system will move out of $z$ only by going back to $x$, when coalition $\{2,3\}$ meets.

Note that the unique Walrasian allocation $w=\left(e_{3}, e_{1}, e_{2}\right)$ also constitutes a singleton recurrent class.

We can prove the following result, characterizing the recurrent classes of the unperturbed process $\mathcal{M}^{0}$ :

Proposition 1 The recurrent classes of the unperturbed process $\mathcal{M}^{0}$ take the following two forms:

(i) Singleton recurrent classes, each of which containing each core allocation.

(ii) Non-singleton recurrent classes: in each of them, the allocations are individually rational but are not core allocations.

Proof: It is clear that each core allocation constitutes an absorbing state of $\mathcal{M}^{0}$, and that every absorbing state must be a core allocation. For the second form of recurrent class,

is the case if only those agents in $N \backslash S$ who are directly or indirectly affected by the reallocation proposed by $S$ are sent to their initial endowments, while the rest of the economy stays put. 
note that, by construction of the system, no state in a recurrent class can ever be nonindividually rational: if at some state $x$ in which $e_{i} \succ_{i} x_{i}$ the coalition $\{i\}$ is chosen, then, the system moves to the individual endowment $e$, never to return to a non-individually rational allocation. It is also clear that each of the states in the recurrent class cannot be absorbing, i.e., a core allocation.

Thus, each core allocation is an absorbing state of the unperturbed Markov process $\mathcal{M}^{0}$, and in principle there may be additional non-singleton recurrent classes, as that in Example 1. Note also that as soon as the economy has more than one core allocation, the system $\mathcal{M}^{0}$ has many stationary distributions.

\section{A Perturbed Recontracting Process}

Next we introduce the perturbed Markov process $\mathcal{M}^{\epsilon}$ for an arbitrary small $\epsilon \in(0,1)$, a perturbation of $\mathcal{M}^{0}$. Suppose the state of the system is the allocation $x$ and that coalition $S$ meets. We shall say that a member of $S$ makes a "mistake" when he signs a contract that either leaves him indifferent to the same house he already had or he becomes worse off upon signing. Each of the members of $S$ may make one of these "mistakes" with a small probability, as a function of $\epsilon>0$, independently of the others. Specifically, for a small fixed $\epsilon \in(0,1)$, we shall postulate that an agent's probability of agreeing to a new allocation that leaves him indifferent is $\epsilon$, while the probability of agreeing to an allocation that makes him worse off is $\epsilon^{\lambda}$ for a sufficiently large positive integer $\lambda$. That is, the latter mistakes are much less likely than the former, while both are rare events in the agent's decision-making process. $^{7}$

Before we define the perturbed process, we need some notation and definitions. Consider an arbitrary pair of allocations $z$ and $z^{\prime}$. Let $T\left(z, z^{\prime}\right) \subseteq 2^{N} \backslash\{\emptyset\}$ be the set of coalitions such that, if chosen, can induce the perturbed system to transit from $z$ to $z^{\prime}$ in one step. Note

\footnotetext{
${ }^{7}$ Following Bergin and Lipman (1996), the results will depend on the specification of these probabilities. Our results are robust to any specification that preserves the Pareto criterion, i.e., a transition that makes an agent worse off be less likely than one in which the only frictions are indifferences (e.g., a lexicographic specification, in which making an agent worse off implies automatically a lower transition probability than one involving only indifferences).
} 
that it is always the case that $T\left(z, z^{\prime}\right) \neq \emptyset$ since $N \in T\left(z, z^{\prime}\right)$ for any $z$ and $z^{\prime}$.

In the direct transition from $z$ to $z^{\prime}$ and for each $S \in T\left(z, z^{\prime}\right)$, define the following numbers:

$$
\begin{aligned}
n_{I}\left(S, z, z^{\prime}\right) & =\left|\left\{i \in S: z_{i} \sim_{i} z_{i}^{\prime}\right\}\right|, \\
n_{W}\left(S, z, z^{\prime}\right) & =\left|\left\{i \in S: z_{i} \succ_{i} z_{i}^{\prime}\right\}\right|, \\
n\left(S, z, z^{\prime}\right) & =\lambda n_{W}\left(S, z, z^{\prime}\right)+n_{I}\left(S, z, z^{\prime}\right) .
\end{aligned}
$$

Here $n_{I}\left(S, z, z^{\prime}\right)$ is the number of individuals in $S$ that are indifferent between allocations $z$ and $z^{\prime}$. Similarly, $n_{W}\left(S, z, z^{\prime}\right)$ is the number of members of $S$ that make a mistake agreeing to move from $z$ to $z^{\prime}$, which is worse for them. Finally, $n\left(S, z, z^{\prime}\right)$ is the weighted number of mistakes that are made in the transition from $z$ to $z^{\prime}$, where $\lambda>1$ is the weight given to "serious" mistakes relative to those that lead to just indifferences.

In the perturbed Markov process $\mathcal{M}^{\epsilon}$ the transition probabilities are calculated as follows. Suppose that the system is in allocation $z$. All coalitions are chosen with a fixed positive probability. Assume coalition $S$ is chosen. If $S \notin T\left(z, z^{\prime}\right)$, then $S$ moves to $z^{\prime}$ with probability 0 . If $S \in T\left(z, z^{\prime}\right)$ and $n\left(S, z, z^{\prime}\right)>0$, then coalition $S$ agrees to move to $z^{\prime}$ with probability $\epsilon^{n\left(S, z, z^{\prime}\right)}$. If $S \in T\left(z, z^{\prime}\right)$ and $n\left(S, z, z^{\prime}\right)=0$, coalition $S$ moves to those $z^{\prime}$ with some (possibly state-dependent) probability $\delta$, where $0<\delta<\epsilon /|A|$.

For all $\epsilon \in(0,1)$ small enough, the system $\mathcal{M}^{\epsilon}$ is a well-defined irreducible Markov process. As such, it has a unique invariant distribution. This distribution gives the probability that the system is in each of the allocations in the long run. We are interested in the limit of the corresponding invariant distributions as $\epsilon$ tends to 0. More precisely, we are interested in the allocations that are assigned positive probability by this limiting distribution. These allocations are called the stochastically stable allocations.

In order to obtain our results, we will use the techniques developed by Kandori, Mailath and Rob (1993), and Young (1993). But before that, we need some definitions.

Note that by the definition of the perturbed Markov process $\mathcal{M}^{\epsilon}$, for every two allocations $z$ and $z^{\prime}$, the direct transition probability $\mu_{z, z^{\prime}}(\epsilon)$ converges to the limit transition probability $\mu_{z, z^{\prime}}(0)$ of the unperturbed process $\mathcal{M}^{0}$ at an exponential rate. In particular, for all allocations $z, z^{\prime}$ such that $\mu_{z, z^{\prime}}=0$, the convergence is at a rate $r\left(z, z^{\prime}\right)=$ $\min _{S \in T\left(z, z^{\prime}\right)} \lambda n_{W}\left(S, z, z^{\prime}\right)+n_{I}\left(S, z, z^{\prime}\right)$. We call the value $r\left(z, z^{\prime}\right)$ the resistance of the direct 
transition from allocation $z$ to allocation $z^{\prime}$. For any two allocations $z, z^{\prime}$, a $\left(z, z^{\prime}\right)$-path is a sequence of allocations $\xi=\left(i_{0}, i_{1}, \ldots, i_{m}\right)$ such that $i_{0}=z, i_{m}=z^{\prime}$. The resistance of the path $\xi$ is the sum of the resistances of its transitions. Let $Z^{0}=\left\{E^{0}, E^{1}, \ldots, E^{Q}\right\}$ be the set of recurrent classes of the unperturbed process $\mathcal{M}^{0}$, and consider the complete directed graph with vertex set $Z^{0}$. We want to define the resistance of each one of the edges in this graph. For this, let $E^{i}$ and $E^{j}$ be two elements of $Z^{0}$. The resistance of the edge $\left(E^{i}, E^{j}\right)$ in the graph, $r\left(E^{i}, E^{j}\right)$, is the minimum resistance over all the resistances of the $\left(z^{i}, z^{j}\right)$-paths, where $z^{i} \in E^{i}$ and $z^{j} \in E^{j}$. A spanning tree rooted at $E^{j}$, or $E^{j}$-tree, is a set of directed edges such that from every recurrent class different from $E^{j}$, there is a unique directed path in the tree to $E^{j}$. The resistance of a spanning tree rooted at $E^{j}$ is the sum of the resistances of its edges. The stochastic potential of the recurrent class $E^{j}$ is the minimum resistance attained by a spanning tree rooted at $E^{j}$. As shown in Young (1993), the set of stochastically stable states of the perturbed process $\mathcal{M}^{\epsilon}$ consists of those states belonging to the recurrent classes with minimum stochastic potential.

\section{Economies with Singleton Indifference Sets}

In this section we shall assume that for every agent $i \in N$ the preference relation $\succeq_{i}$ is antisymmetric, which implies that all indifference sets are singletons. Making this assumption, Roth and Postlewaite (1977) proved the following result:

Lemma 1 Let $\mathcal{E}$ be a housing economy where all preferences are strict. Then,

(i) There is a unique Walrasian allocation $w$.

(ii) The allocation $w$ is the only strong core allocation.

(iii) For every allocation $x \in A, x \neq w$, there exists a coalition $S$ such that $w_{S}$ is feasible for $S$ and satisfies $w_{i} \succeq_{i} x_{i}$ for all $i \in S$ and $w_{j} \succ_{j} x_{j}$ for some $j \in S$.

Lemma 1 will be useful in proving our first main result, to which we turn now.

Theorem 1 Let $\mathcal{E}$ be a housing economy where all preferences are strict. Suppose that $\lambda>|N|-2$. Then, the unique stochastically stable allocation of the perturbed process $\mathcal{M}^{\epsilon}$ is the Walrasian allocation $w$. 
Proof: Since when $|N|=2$, the core consists of the singleton $w$ and there are only two feasible allocations, it is clear that the statement holds, by Proposition 1. Thus, assume that $|N| \geq 3$. Recall that we denote the set of recurrent classes of the unperturbed process $\mathcal{M}^{0}$ by $\left\{E^{0}, E^{1}, \ldots, E^{Q}\right\}$, and let $E^{0}$ be the singleton recurrent class containing the Walrasian allocation $w$. Denote the stochastic potential of $E^{0}$ by $\operatorname{sp}\left(E^{0}\right)$. We show that the stochastic potential of any other recurrent class $E^{k}, k=1, \ldots, Q$, is greater than $\operatorname{sp}\left(E^{0}\right)$. Let $E^{k} \neq$ $E^{0}$ be an arbitrary recurrent class. Consider an $E^{k}$-tree of stochastic potential $\operatorname{sp}\left(E^{k}\right)$. Introduce in it the following two modifications:

(i) Delete the edge that connects the class $E^{0}$ to its successor $E^{j}$ on the path to $E^{k}$.

(ii) Add a directed edge going from $E^{k}$ to $E^{0}$.

Note that the resulting graph is an $E^{0}$-tree. Moreover, the resistance of this new $E^{0}$-tree $r(T)$ equals

$$
r(T)=\operatorname{sp}\left(E^{k}\right)-r\left(E^{0}, E^{j}\right)+r\left(E^{k}, E^{0}\right) .
$$

To finish the proof, we show in the following two lemmas that $r\left(E^{0}, E^{j}\right)>r\left(E^{k}, E^{0}\right)$. This means that we would have constructed an $E^{0}$-tree whose resistance is less than $\operatorname{sp}\left(E^{k}\right)$, thereby showing that $\operatorname{sp}\left(E^{0}\right)<\operatorname{sp}\left(E^{k}\right)$.

Lemma 2 Consider the edge $E^{0} \rightarrow E^{j}$ that is deleted from the $E^{k}$-tree. Then, $r\left(E^{0}, E^{j}\right) \geq$ $\lambda$.

Proof: Consider a path that attains the resistance $r\left(E^{0}, E^{j}\right)$, of the edge that connects the Walrasian allocation, $w$, to the recurrent class $E^{j}$, and let $x^{1}$ be the first allocation in this path. We claim that, in the direct transition from $w$ to $x^{1}$, at least one agent becomes worse off. Let the coalition involved in this transition be $S^{1}$. If it were the case that $x_{i}^{1} \succeq_{i} w_{i}$ for every $i \in S^{1}$, we would be saying that $w$ is not a strong core allocation, contradicting Lemma 1, part (ii). Therefore, at least one agent becomes worse off in this direct transition, from which it follows that $r\left(E^{0}, E^{j}\right) \geq \lambda$.

Lemma 3 Consider the edge $E^{k} \rightarrow E^{0}$ that is added to the $E^{k}$-tree. Then, $r\left(E^{k}, E^{0}\right) \leq$ $|N|-2$. 
Proof: We calculate an upper bound for $r\left(E^{k}, E^{0}\right)$ as follows. Let $x \in E^{k}$. By Lemma 1, part (iii), there exists a coalition $S$ such that $\left(w_{i}\right)_{i \in S} \in A_{S}, w_{i} \succeq_{i} x_{i}$ for all $i \in S$ and $w_{j} \succ_{j} x_{j}$ for some $j \in S$. In the next paragraphs, we refer to $S$ as one of the maximal (in the sense of set inclusion) such coalitions. We have two cases.

Case 1: $S=N$. In this case, the maximum possible resistance associated with the direct transition from $x$ to $w$ is $(|N|-2)$, i.e., the one given by the highest number of indifferences that can occur in $N$.

Case 2: $S \neq N$. This case admits two subcases:

Subcase 2.1: Suppose that $x_{-S} \notin A_{-S}$. Then, when coalition $S$ meets, the system moves to $y=\left(w_{S}, e_{-S}\right)$ with positive probability. The resistance of this transition cannot be greater than $(|N|-2)$ because, within $S$, one can have at most $(|S|-2)$ indifferences. But note that from $y$, the system can move to $w$ with a resistance no bigger than $|N \backslash S|-2$ : if necessary, the coalition $T \subseteq N \backslash S$ of agents who are not receiving their Walrasian house at $y$ will be partitioned in subsets (according to the trading cycles), each of which to perform the necessary trade so that the final result is $w$. Therefore, since $|S| \leq|N|-2$, the number of indifferences found in this transition is at most $(|N|-4)$.

Subcase 2.2: $x_{-S} \in A_{-S}$. In this case, coalition $S$ meets and the system moves to $y=\left(w_{S}, x_{-S}\right)$ with positive probability. But then, by our choice of $S$ and Lemma 1, part (iii) applied to the subeconomy consisting of agents $N \backslash S$, it must necessarily be the case that $x_{-S}=w_{-S}$. Therefore, $N \backslash S=\emptyset$ because otherwise $S$ would not be a maximal blocking coalition. So in this case $S=N$ and we are back in case 1 .

Therefore, the resistance of the transition $E^{k} \rightarrow E^{0}$ is bounded above by the maximum of the two expressions involved in the two cases analyzed, which is $(|N|-2)$.

In consequence, it follows from our assumption on the size of $\lambda$ that $r\left(E^{k}, E^{0}\right)<$ $r\left(E^{0}, E^{j}\right)$, which concludes the proof.

\section{Economies with Non-Singleton Indifference Sets}

In this section we explore how the stochastic process of recontracting with mistakes, $\mathcal{M}^{\epsilon}$, performs over the class of economies that allow non-singleton indifference sets for some agents. Over this larger class of economies, recall that Proposition 1 still holds. However, 
the conclusions of Lemma 1 do not extend. First, although existence is guaranteed, there may be multiple Walrasian allocations. Second, the strong core may also contain multiple allocations, while it may sometimes be empty. And third, the "global dominance" property of Walrasian allocations as specified in Lemma 1, part (iii), is also lost.

In economies with only strict preferences, the Walrasian allocation correspondence and the strong core coincide, and under our assumption on $\lambda$, the same allocation is the only one that passes the test of stochastic stability. It is convenient, therefore, to examine the larger class of economies to disentangle the different forces at work in the selection of the stochastically stable allocations. The recontracting system with mistakes gives rise to complicated dynamics, and no general result of equivalence can be established. Therefore, we learn that the paths of least resistance followed in our stochastic dynamic analysis are not intrinsically associated with the strong core property or with the Walrasian property of allocations.

We shall present four examples. We arrange them by increasing difficulty and relevance. Indeed, we regard Example 5 as the other main result of the paper. In some of these examples, one agent has a completely flat indifference map, but this is only for simplicity of exposition. Also, in the examples we shall use the notation $z \rightarrow_{S}^{r} z^{\prime}$ to express that the transition of least resistance from $z$ to $z^{\prime}$ takes place through coalition $S$ at a resistance $r$.

We begin by showing that the set of stochastically stable allocations is not the strong core. As we just pointed out, the strong core may be empty in these economies, while stochastic stability always selects at least one allocation; but even when the strong core is non-empty, one can generate examples where it does not coincide with the set of stochastically stable states of $\mathcal{M}^{\epsilon}$.

Example 2 In this example, a non-empty strong core is strictly contained in the set of stochastically stable allocations. Let $N=\{1,2\}$ and agents' preferences be described as follows:

$$
\begin{aligned}
& e_{1} \sim_{1} e_{2} ; \\
& e_{1} \succ_{2} e_{2} .
\end{aligned}
$$

In this economy there are two allocations, both of which are Walrasian, but only the one resulting from trade is in the strong core. Note that both allocations are stochastically stable: $\left(e_{1}, e_{2}\right) \rightarrow_{N}^{1}\left(e_{2}, e_{1}\right)$ and $\left(e_{2}, e_{1}\right) \rightarrow_{\{1\}}^{1}\left(e_{1}, e_{2}\right)$. 
The next example shows that the set of stochastically stable allocations may be a strict subset of the strong core and of the set of Walrasian allocations.

Example 3 Let $N=\{1,2,3\}$ and the agents' preferences be given by:

$$
\begin{aligned}
& e_{1} \sim_{1} e_{2} \sim_{1} e_{3} \\
& e_{1} \sim_{2} e_{3} \succ_{2} e_{2} \\
& e_{1} \sim_{3} e_{2} \succ_{3} e_{3} .
\end{aligned}
$$

In this economy, all allocations except the initial endowment allocation are Walrasian and belong to the core. The strong core consists of the following three allocations: $\left(e_{2}, e_{3}, e_{1}\right)$, $\left(e_{3}, e_{1}, e_{2}\right)$ and $\left(e_{1}, e_{3}, e_{2}\right)$. The unique stochastically stable allocation is $x=\left(e_{1}, e_{3}, e_{2}\right)$. To see that $x$ is the only allocation with minimum stochastic potential, one can construct an $x$-tree as follows. First, we note that the only recurrent classes of $\mathcal{M}^{0}$ are the five absorbing states corresponding to each Walrasian allocation. Next, note that to go from $\left(e_{2}, e_{3}, e_{1}\right)$ to $x$ can be done with a resistance of 1 (only one indifference): $\left(e_{2}, e_{3}, e_{1}\right) \rightarrow_{\{1\}}^{1}\left(e_{1}, e_{2}, e_{3}\right) \rightarrow_{\{2,3\}}^{0}$ $x$. The same goes for the transition $\left(e_{3}, e_{1}, e_{2}\right)$ to $x:\left(e_{3}, e_{1}, e_{2}\right) \rightarrow_{\{1\}}^{1}\left(e_{1}, e_{2}, e_{3}\right) \rightarrow_{\{2,3\}}^{0} x$. As for the other transitions, we have $\left(e_{3}, e_{2}, e_{1}\right) \rightarrow_{\{2,3\}}^{1} x$ and $\left(e_{2}, e_{1}, e_{3}\right) \rightarrow_{\{2,3\}}^{1} x$. Therefore, the resistance of this $x$-tree is 4 and one cannot build a cheaper tree than that. On the other hand, to get out of $x$, the resistance will always be at least 2 , i.e., at least two indifferences, which implies that, in constructing a tree for any of the other recurrent classes, its resistance must be at least 5 .

Our next example illustrates the only substantive difference in results between the recontracting process $\mathcal{M}^{\epsilon}$ and its counterpart based on weak recontracting that will be studied in Section 7. Specifically, we now construct an economy for which all recurrent classes of $\mathcal{M}^{0}$ are singletons and where the set of stochastically stable allocations of $\mathcal{M}^{\epsilon}$ contains an allocation that is not Walrasian.

Example 4 Let $N=\{1,2,3,4\}$ and the agents' preferences be described as follows: 


$$
\begin{aligned}
& e_{1} \sim_{1} e_{3} \sim_{1} e_{4} \succ_{1} e_{2} ; \\
& e_{1} \sim_{2} e_{4} \succ_{2} e_{3} \succ_{2} e_{2} ; \\
& e_{2} \succ_{3} e_{1} \succ_{3} e_{3} \succ_{3} e_{4} ; \\
& e_{2} \succ_{4} e_{3} \succ_{4} e_{4} \succ_{4} e_{1} .
\end{aligned}
$$

One can check that there are four Walrasian allocations: $w_{1}=\left(e_{3}, e_{4}, e_{1}, e_{2}\right), w_{2}=\left(e_{1}, e_{4}, e_{3}, e_{2}\right)$, $w_{3}=\left(e_{4}, e_{1}, e_{3}, e_{2}\right)$, and $w_{4}=\left(e_{3}, e_{1}, e_{2}, e_{4}\right)$. In addition, there are three other core allocations that are not Walrasian: $y_{1}=\left(e_{1}, e_{4}, e_{2}, e_{3}\right), y_{2}=\left(e_{4}, e_{1}, e_{2}, e_{3}\right)$, and $y_{3}=\left(e_{4}, e_{3}, e_{1}, e_{2}\right)$. Since Proposition 1 also applies to the economies considered in this section, it follows that each of these seven allocations constitutes an absorbing state of $\mathcal{M}^{0}$. Further, it can be checked that there are no additional recurrent classes. Apart from the seven core allocations, the only absorbing states of $\mathcal{M}^{0}$, there are four more individually rational allocations. One is $e=\left(e_{1}, e_{2}, e_{3}, e_{4}\right)$-the initial endowment allocation-, and the other three are $x_{1}=\left(e_{1}, e_{3}, e_{2}, e_{4}\right), x_{2}=\left(e_{3}, e_{2}, e_{1}, e_{4}\right)$ and $x_{3}=\left(e_{4}, e_{2}, e_{1}, e_{3}\right)$. It can be checked that from each of these four allocations one can go at zero resistance to one of the core allocations.

Consider now the following $\left\{y_{1}\right\}$-tree comprising the following edges: $w_{1} \rightarrow y_{1}, w_{2} \rightarrow w_{1}$, $w_{3} \rightarrow w_{1}, w_{4} \rightarrow w_{2}, y_{2} \rightarrow w_{2}, y_{3} \rightarrow y_{1}$. We check next that each of these edges has resistance 1 (corresponding to a transition with only one indifference), thereby implying that the tree has minimum stochastic potential. We detail these transitions at present:

$$
\begin{gathered}
w_{1} \rightarrow_{\{1\}}^{1} e \rightarrow_{\{2,3,4\}}^{0} y_{1}, \\
w_{2} \rightarrow_{\{1,3\}}^{1} x_{2} \rightarrow_{\{2,4\}}^{0} w_{1}, \\
w_{3} \rightarrow_{\{1,3\}}^{1} x_{2} \rightarrow_{\{2,4\}}^{0} w_{1}, \\
w_{4} \rightarrow_{\{2,4\}}^{1} w_{2}, \\
y_{2} \rightarrow_{\{2,4\}}^{1} w_{2}, \\
y_{3} \rightarrow_{\{1\}}^{1} e \rightarrow_{\{2,3,4\}}^{0} y_{1} .
\end{gathered}
$$

Thus, $y_{1}$ is stochastically stable, although it is not a Walrasian allocation. It is not in the strong core either: indeed, the unique strong core allocation in this economy is $w_{1}$. 
The next example shows how different the conclusions one reaches in the analysis of cooperation with mistakes may be from those of standard cooperative game theory. It turns out that in a dynamic model where agents may make mistakes in decision-making, the core may not agree with the set of states that are visited by the process a positive proportion of time. In contrast, some non-core allocations may fare better in this sense than some core allocations.

Example $5^{8}$ This example, an outgrowth of Example 1, shows that a cycle of non-core allocations may be stochastically stable, at the same time as some core allocations having higher stochastic potential. Let $N=\{1,2,3,4\}$, and the agents' preferences be as follows:

$$
\begin{aligned}
& e_{4} \succ_{1} e_{3} \succ_{1} e_{2} \succ_{1} e_{1} ; \\
& e_{1} \succ_{2} e_{3} \succ_{2} e_{2} \succ_{2} e_{4} ; \\
& e_{2} \succ_{3} e_{1} \succ_{3} e_{3} \succ_{3} e_{4} ; \\
& e_{1} \sim_{4} e_{2} \sim_{4} e_{3} \sim_{4} e_{4} .
\end{aligned}
$$

Consider the following three allocations: $x=\left(e_{1}, e_{3}, e_{2}, e_{4}\right), y=\left(e_{2}, e_{1}, e_{3}, e_{4}\right)$ and $z=\left(e_{3}, e_{2}, e_{1}, e_{4}\right)$. Since agent 4 cannot strictly improve, he cannot be part of any blocking coalition. In fact, as in Example 1, these three allocations constitute a recurrent class: if the system is at $x$, the state changes only when coalition $\{1,2\}$ meets, yielding $y$. At $y$, the system can move only to $z$, when coalition $\{1,3\}$ meets. Finally, the system will move out of $z$ only by going back to $x$, when coalition $\{2,3\}$ meets. That is, $x \rightarrow_{\{1,2\}}^{0} y \rightarrow_{\{1,3\}}^{0} z \rightarrow_{\{2,3\}}^{0} x$.

The core consists of the following five allocations: $c_{1}=\left(e_{3}, e_{1}, e_{2}, e_{4}\right), c_{2}=\left(e_{4}, e_{1}, e_{2}, e_{3}\right)$, $c_{3}=\left(e_{4}, e_{1}, e_{3}, e_{2}\right), c_{4}=\left(e_{4}, e_{3}, e_{1}, e_{2}\right)$, and $c_{5}=\left(e_{4}, e_{3}, e_{2}, e_{1}\right)$. It is easy to see that these five absorbing states -i.e., the core allocations- and the cycle are the only recurrent classes of the unperturbed system: the 12 allocations where $e_{4}$ is allocated to either agent 2 or agent 3 are not even individually rational. And from each of the remaining four allocations, one gets to one of the already identified recurrent classes with 0 resistance:

\footnotetext{
${ }^{8}$ As communicated to us by Bob Aumann, a similar story is told in the Talmud: there is a cycle of three two-person coalitions improving the status quo. The three players involved in the cycle are the two wives of a man and a third party who buys the man's estate. The cycle occurs after the man died and left his estate. The diseased agent naturally corresponds to our agent 4 , who has a flat indifference map. See also Binmore (1985) for a more recent related problem.
} 
$a_{1}=\left(e_{1}, e_{2}, e_{3}, e_{4}\right) \rightarrow_{\{1,2,3\}}^{0} c_{1}, a_{2}=\left(e_{2}, e_{3}, e_{1}, e_{4}\right) \rightarrow_{\{1,2,3\}}^{0} c_{1}, a_{3}=\left(e_{4}, e_{2}, e_{1}, e_{3}\right) \rightarrow_{\{2,3\}}^{0} x$, and $a_{4}=\left(e_{4}, e_{2}, e_{3}, e_{1}\right) \rightarrow_{\{2,3\}}^{0} c_{5}$.

Let $E=\{x, y, z\}$ be the non-singleton recurrent class consisting of non-core allocations. Next, we construct an E-tree and show that it has minimum stochastic potential. This tree must have five edges, coming out of each of the five core allocations. We detail the transitions below:

$$
\begin{gathered}
c_{1} \rightarrow_{\{1,4\}}^{1} a_{4} \rightarrow_{\{2,3\}}^{0} c_{5}, \\
c_{j} \rightarrow_{\{4\}}^{1} a_{1} \rightarrow_{\{1,2\}}^{0} y, \quad \text { for } j=2,3,4,5 .
\end{gathered}
$$

Therefore, the class $E$ has minimum stochastic potential.

Note also that there are four Walrasian allocations: $c_{1}, c_{2}, c_{3}$ and $c_{5}$. Thus, the example also shows that there are non-Walrasian stochastically stable allocations.

However, apart from $E$, the only stochastically stable allocations are the four Walrasian allocations $c_{1}, c_{2}, c_{3}$ and $c_{5}$ : there are allocations in the core that are not visited in the long run but a zero proportion of time. In particular, it can be checked that it takes at least two indifferences to get out of other recurrent classes to go to $c_{4}$. This implies that $c_{4}$ cannot be stochastically stable. As an illustration, we construct a $\left\{c_{4}\right\}$-tree as follows:

$$
\begin{gathered}
c_{1} \rightarrow_{\{1,4\}}^{1} a_{4} \rightarrow_{\{2,3\}}^{0} c_{5}, \\
c_{j} \rightarrow_{\{4\}}^{1} a_{1} \rightarrow_{\{1,2\}}^{0}(y \in E), \quad \text { for } j=2,3,5, \\
(z \in E) \rightarrow{ }_{N}^{2} c_{4} .
\end{gathered}
$$

Thus, the dynamic analysis, which sheds light on how each allocation comes about, uncovers a difference among core allocations. Some core allocations (like $c_{4}$ in the example) are actually very hard to get to, when compared to other allocations in the core or even outside of the core. The point is that the usual emphasis in the definition of the core is given to the non-existence of a coalitional blocking move, whereas the issue of how the allocation in question comes about is disregarded. Dynamics should have something to say about the matter, and Example 5 is a confirmation that this is indeed the case. 


\section{Recontracting Based on Weak Blocking}

As a robustness check of our results, we analyze in this section a Markov process in which transitions take place whenever there is an instance of weak coalitional blocking, instead of the strict version thereof, as was the case in the process $\mathcal{M}^{0}$.

\subsection{The Unperturbed Process}

Consider the following unperturbed Markov process $\mathcal{M}_{W}^{0}$, which represents the "weak recontracting" version of the process presented in Section 3. In each period $t$, if the system is at the allocation $x(t)$, all coalitions are chosen with arbitrary, but positive, probability. Suppose coalition $S$ is chosen.

(i) If there exists an $S$-allocation $y_{S} \in A_{S}$ such that $y_{i} \succeq_{i} x_{i}(t)$ for all $i \in S$ and $y_{j} \succ_{j} x_{j}(t)$ for some $j \in S$, the coalition moves with positive probability to some of such $y_{S}$ in that period. Then, the new state is either

$$
\begin{aligned}
& x(t+1)=\left(y_{S}, x_{-S}(t)\right) \text { if } x_{-S}(t) \in A_{-S}, \text { or } \\
& x(t+1)=\left(y_{S}, e_{-S}\right) \text { if } x_{-S}(t) \notin A_{-S} .
\end{aligned}
$$

(ii) Otherwise, $x(t+1)=x(t)$.

The interpretation of this new process is one of weak coalitional recontracting. Following a status quo, a coalition can form and modify the status quo if at least one member of the coalition improves as a result, and none of the other members is made worse off. Apart from this change, the process is identical to the one in Section 3. That is, when the weak coalitional recontracting move happens, upon coalition $S$ forming, the complement coalition $N \backslash S$ continues to have the same houses as before if this is feasible for them. Otherwise, $N \backslash S$ breaks apart and each of the agents in it receives his individual endowment (the same comment as in footnote 6 applies here in terms of alternative specifications). If after coalition $S$ gets together, it cannot find any such weak coalitional improvement, the original status quo persists.

The absorbing states of the unperturbed process $\mathcal{M}_{W}^{0}$ are precisely the strong core allocations of the economy. However, the absorbing states are not the only recurrent classes 
of $\mathcal{M}_{W}^{0}$. The easiest way to see this is to note, as we already did, that there are economies in which the strong core is empty.

We can prove the following result, similar to Proposition 1, characterizing the recurrent classes of the unperturbed process $\mathcal{M}_{W}^{0}$ :

Proposition 2 The recurrent classes of the unperturbed process $\mathcal{M}_{W}^{0}$ take the following two forms:

(i) Singleton recurrent classes, each of which containing each strong core allocation.

(ii) Non-singleton recurrent classes: in each of them, the allocations are individually rational but are not strong core allocations.

Proof: The proof is similar to that of Proposition 1.

Thus, each strong core allocation is an absorbing state of the unperturbed Markov process $\mathcal{M}_{W}^{0}$, and in principle there may be additional non-singleton recurrent classes. Note also that as soon as the economy has more than one strong core allocation, the system $\mathcal{M}_{W}^{0}$ has many stationary distributions. The conclusions of Proposition 2 extend to all economies where preferences are complete and transitive, not necessarily antisymmetric.

One can now obtain a simple generalization of the conclusions of Theorem 1 . In fact, the Walrasian result is obtained even before mistakes are added to the process.

Proposition 3 Let $\mathcal{E}$ be a housing economy where all preferences are strict. Then, the unperturbed process $\mathcal{M}_{W}^{0}$ is ergodic and the only recurrent class is the singleton consisting of the unique Walrasian allocation.

Proof: The proof follows from the definition of the transitions in the process and from Lemma 1.

Notice how, as in Theorem 1, the "global dominance" property of the Walrasian allocation in these economies makes it the only prediction of the dynamic process, this time as the strong attractor of the unperturbed system. 


\section{$7.2 \quad$ A Perturbed Process}

Our next attempt is the generalization of the non-Walrasian conclusions obtained by our dynamic analysis in economies with indifferences. For this purpose, we next introduce the perturbed Markov process $\mathcal{M}_{W}^{\epsilon}$ for an arbitrary small $\epsilon \in(0,1)$, a perturbation of $\mathcal{M}_{W}^{0}$. Suppose the state of the system is the allocation $x$ and that coalition $S$ meets. We shall say that a member of $S$ makes a "mistake" when he signs a contract that makes him worse off upon signing. Each of the members of $S$ may make a "mistake" with a small probability, as a function of $\epsilon>0$, independently of the others. Specifically, for a small fixed $\epsilon \in(0,1)$, we shall postulate that an agent's probability of agreeing to an allocation that makes him worse off is $\epsilon$. In addition, we shall say that the coalition $S$ makes an "indifference-based coalitional mistake" if it agrees to a trade which leaves each and every one of its members exactly indifferent. In consonance with our assumption on $\lambda$ made in Section 4 (see footnote 7), indifference-based coalitional mistakes are somewhat less serious than individual mistakes. On the other hand, they will be more unlikely the larger the size of the coalition involved. Details are provided below.

Before we define the perturbed process, we need some notation and definitions. Consider an arbitrary pair of allocations $z$ and $z^{\prime}$. Let $T\left(z, z^{\prime}\right) \subseteq 2^{N} \backslash\{\emptyset\}$ be the set of coalitions such that, if chosen, can induce the system to transit from $z$ to $z^{\prime}$ in one step. Again, note that it is always the case that $T\left(z, z^{\prime}\right) \neq \emptyset$ since $N \in T\left(z, z^{\prime}\right)$ for any $z$ and $z^{\prime}$.

In the direct transition from $z$ to $z^{\prime}$ and for each $S \in T\left(z, z^{\prime}\right)$, define the following numbers:

$$
\begin{aligned}
n_{I}\left(S, z, z^{\prime}\right) & =\left|\left\{i \in S: z_{i} \sim_{i} z_{i}^{\prime}\right\}\right|, \\
n_{W}\left(S, z, z^{\prime}\right) & =\left|\left\{i \in S: z_{i} \succ_{i} z_{i}^{\prime}\right\}\right|
\end{aligned}
$$

In the perturbed Markov process $\mathcal{M}_{W}^{\epsilon}$ the transition probabilities are calculated as follows. Suppose that the system is in allocation $z$. All coalitions are chosen with a fixed positive probability. Assume coalition $S$ is chosen. If $S \notin T\left(z, z^{\prime}\right)$, then the system moves to $z^{\prime}$ with probability 0 . If $S \in T\left(z, z^{\prime}\right)$ and $n_{W}\left(S, z, z^{\prime}\right)>0$, then coalition $S$ agrees to move to $z^{\prime}$ with probability $\epsilon^{n_{W}\left(S, z, z^{\prime}\right)}$. If $S \in T\left(z, z^{\prime}\right), n_{W}\left(S, z, z^{\prime}\right)=0$, and $n_{I}\left(S, z, z^{\prime}\right)=|S|$ 
coalition $S$ moves to $z^{\prime}$ with probability $\epsilon^{|S| /|N|}$. Finally, if $S \in T\left(z, z^{\prime}\right), n_{W}\left(S, z, z^{\prime}\right)=0$, and $n_{I}\left(S, z, z^{\prime}\right)<|S|$, coalition $S$ moves to $z^{\prime}$ with some (possibly state-dependent) probability $\delta$, where $0<\delta<\epsilon /|A|$.

For all $\epsilon \in(0,1)$ small enough, the system $\mathcal{M}_{W}^{\epsilon}$ is a well-defined irreducible Markov process. Therefore, it has a unique invariant distribution $\mu_{W}^{\epsilon}$. We are now interested in identifying the stochastically stable states of $\mathcal{M}_{W}^{\epsilon}$, i.e., those allocations in the support of $\lim _{\epsilon \rightarrow 0} \mu_{W}^{\epsilon}$. Of course, we already know the answer for economies with no indifferences: from Proposition 3, stochastic stability, which always selects a non-empty set of states, yields the unique Walrasian allocation. The rest of the section considers economies with indifferences.

Note that by the definition of the perturbed Markov process $\mathcal{M}_{W}^{\epsilon}$, for every two allocations $z$ and $z^{\prime}$, the direct transition probability $\mu_{z, z^{\prime}}(\epsilon)$ converges to the limit transition probability $\mu_{z, z^{\prime}}(0)$ of the unperturbed process $\mathcal{M}_{W}^{0}$ at an exponential rate. In particular, if we let

$$
r\left(S, z, z^{\prime}\right)= \begin{cases}n_{W}\left(S, z, z^{\prime}\right) & \text { if } n_{W}\left(S, z, z^{\prime}\right)>0 \\ |S| /|N| & \text { if } n_{W}\left(S, z, z^{\prime}\right)=0 \text { and } n_{I}\left(S, z, z^{\prime}\right)=|S| \\ \infty & \text { otherwise }\end{cases}
$$

for all allocations $z, z^{\prime}$ such that $\mu_{z, z^{\prime}}=0$, the convergence is at a rate $r\left(z, z^{\prime}\right)=\min _{S \in T\left(z, z^{\prime}\right)} r\left(S, z, z^{\prime}\right)$. As already done in previous sections, we call the value $r\left(z, z^{\prime}\right)$ the resistance of the direct transition from allocation $z$ to allocation $z^{\prime}$. The resistance of a path is defined similarly. Given $Z^{0}=\left\{E^{0}, E^{1}, \ldots, E^{Q}\right\}$, the set of recurrent classes of the unperturbed process $\mathcal{M}_{W}^{0}$, one considers the complete directed graph with vertex set $Z^{0}$, and defines the resistance of each edge in the graph as before. Given a spanning tree rooted at $E^{j}$, one can calculate its stochastic potential. The stochastically stable states of the process $\mathcal{M}_{W}^{\epsilon}$ are those contained in the recurrent classes of $\mathcal{M}_{W}^{0}$ with minimum stochastic potential. As such, these are the allocations that are easiest to access from anywhere else in the system, the only ones in which the system will spend a positive proportion of time in the long run when mistakes are possible but unlikely events.

Now we shall show that suitable generalizations of our previous results are obtained. It will be instructive to separate the analysis into two: economies in which the recurrent classes of $\mathcal{M}_{W}^{0}$ are all singletons, and those in which non-singleton recurrent classes also exist. As 
it turns out, in the former we find the only substantive difference in results between the strict and weak processes of recontracting.

\subsubsection{Economies without Non-Singleton Recurrent Classes}

For this case, stochastic stability will select a subset of the strong core, which is a subset of the Walrasian allocations. Therefore, one can see this as a difference with respect to the results of the process $\mathcal{M}^{\epsilon}$ based on strict recontracting -recall Example 4 (even though the economies where recurrent classes of $\mathcal{M}^{0}$ are all singletons do not coincide with those in which the same is true for $\left.\mathcal{M}_{W}^{0}\right)$. Specifically, one can show the following result.

Proposition 4 Let $\mathcal{E}$ be a housing economy in which all recurrent classes of $\mathcal{M}_{W}^{0}$ are singletons. The stochastically stable states of $\mathcal{M}_{W}^{\epsilon}$ are Walrasian allocations.

Proof: By Proposition 2, if all recurrent classes of the unperturbed process $\mathcal{M}_{W}^{0}$ are singletons, they correspond to each of the strong core allocations. To finish the proof, we show now that all the strong core allocations are Walrasian. To see this, take a strong core allocation $x$ of an economy $\mathcal{E}=\left\langle N, H,\left(\succeq_{i}, e_{i}\right)_{i \in N}\right\rangle$ with indifferences, and consider an economy $\mathcal{E}^{\prime}=\left\langle N, H,\left(\succeq_{i}^{\prime}, e_{i}\right)_{i \in N}\right\rangle$ that is obtained from $\mathcal{E}$ by undoing indifferences in the following way: if for agent $i \in N, x_{i} \sim_{i} h$, then $x_{i} \succ_{i}^{\prime} h$; on the other hand, for all $i \in N$ if $h^{\prime} \succ_{i} h, h^{\prime} \succ_{i}^{\prime} h$. In the resulting economy $\mathcal{E}^{\prime}, x$ is a strong core allocation. To see this, note that if coalition $S$ can weakly block $x$ in the economy $\mathcal{E}^{\prime}$, it could also block in the economy $\mathcal{E}$, contradicting that $x$ is in the strong core of $\mathcal{E}$. Since $\mathcal{E}^{\prime}$ is an economy with no indifferences, by Lemma 1, parts (i) and (ii), $x$ is the only strong core allocation and the only Walrasian allocation of $\mathcal{E}^{\prime}$. But then $x$ must be Walrasian in $\mathcal{E}$, because the same prices that support it in $\mathcal{E}^{\prime}$ also support it in $\mathcal{E}$.

Thus, in economies where all recurrent classes of $\mathcal{M}_{W}^{0}$ are singletons, stochastic stability will select a subset, always non-empty, of the strong core, and therefore of the set of Walrasian allocations. In particular, note how Proposition 3 follows from this result.

The next example shows that the set of stochastically stable allocations of $\mathcal{M}_{W}^{\epsilon}$ may be a strict subset of the strong core (and a fortiori, of the set of Walrasian allocations). 
Example 6 This example studies the process $\mathcal{M}_{W}^{\epsilon}$ on the same economy as in Example 3. That is, let $N=\{1,2,3\}$ and the agents' preferences be given by:

$$
\begin{aligned}
& e_{1} \sim_{1} e_{2} \sim_{1} e_{3} ; \\
& e_{1} \sim_{2} e_{3} \succ_{2} e_{2} ; \\
& e_{1} \sim_{3} e_{2} \succ_{3} e_{3} .
\end{aligned}
$$

Recall that in this economy, all allocations except the initial endowment allocation are Walrasian, and that the strong core consists of the following three allocations: $\left(e_{2}, e_{3}, e_{1}\right)$, $\left(e_{3}, e_{1}, e_{2}\right)$ and $\left(e_{1}, e_{3}, e_{2}\right)$. In each of these allocations, each agent receives one of his topranked houses. Further, each of these allocations Pareto dominates the other three allocations. The three constitute the only recurring classes of the system. However, only $\left(e_{1}, e_{3}, e_{2}\right)$ is stochastically stable in $\mathcal{M}_{W}^{\epsilon}$. The system transits from each of the other two strong core allocations to the endowment with a resistance of only one indifference (that of agent 1) and from there to $\left(e_{1}, e_{3}, e_{2}\right)$ with a resistance of 0 . On the other hand, in order to leave $\left(e_{1}, e_{3}, e_{2}\right)$, the system encounters a resistance of at least 2 : two indifferences in a two-agent coalition containing agent 1 , after which the grand coalition recontracts at no cost leading to one of the other recurrent classes.

\subsubsection{Economies with Non-Singleton Recurrent Classes}

For this class of economies, we shall show that some non-singleton recurrent classes are selected by stochastic stability. When this happens, the weak recontracting process with mistakes identifies extra allocations that the economy will visit a positive proportion of time in the long run, even though none of them are coalitionally stable in the sense of the strong core. The next example shows that the set of stochastically stable states may contain non-Walrasian allocations, as well as allocations that are not Pareto indifferent to Walrasian ones. 
Example 7 Let $N=\{1,2,3\}$ and suppose the agents' preferences are as follows:

$$
\begin{aligned}
& e_{2} \sim_{1} e_{3} \succ_{1} e_{1} ; \\
& e_{1} \succ_{2} e_{3} \succ_{2} e_{2} ; \\
& e_{1} \succ_{3} e_{2} \succ_{3} e_{3} .
\end{aligned}
$$

Here, the strong core is empty because either coalition $\{1,2\}$ or $\{1,3\}$ always improves weakly upon any allocation. There are two Walrasian allocations: $\left(e_{2}, e_{1}, e_{3}\right)$ and $\left(e_{3}, e_{2}, e_{1}\right)$. The endowment allocation and the allocation $\left(e_{1}, e_{3}, e_{2}\right)$ are not in any recurrent class of the unperturbed process based on weak blocking: one cannot get to the endowment from anywhere else by means of weak improvements of any coalition; and one can get to $\left(e_{1}, e_{3}, e_{2}\right)$ in that way only from the endowment allocation. However, the other four allocations constitute a recurrent class. We detail the coalitions involved in each transition:

$$
\left(e_{2}, e_{1}, e_{3}\right) \rightarrow_{N}\left(e_{3}, e_{1}, e_{2}\right) \rightarrow_{\{1,3\}}\left(e_{3}, e_{2}, e_{1}\right) \rightarrow_{N}\left(e_{2}, e_{3}, e_{1}\right) \rightarrow_{\{1,2\}}\left(e_{2}, e_{1}, e_{3}\right)
$$

Therefore, the four allocations in the unique recurrent class are stochastically stable in the process $\mathcal{M}_{W}^{\epsilon}$.

\section{Recontracting with Mistakes and Edgeworth's Tra- dition}

In this section we discuss how our process of recontracting with mistakes integrates in Edgeworth's tradition. In particular, we devote special attention to the issue of when consumption takes place. Our model has several features to be emphasized now:

1. There is a static exchange economy;

2. This economy is repeated over time;

3. In each period a potentially different allocation obtains and, presumably, is consumed. Based on these features, we shall argue that our model is a faithful description, though not the only one, of the recontracting process envisioned by Edgeworth. 
When Edgeworth describes the process of contracting and recontracting, it is clear that he has a static exchange economy in mind:

...the disposition and circumstances of the parties are assumed to remain throughout constant. [Edgeworth (1925), p. 313.]

In this context, the recontracting process consists of a series of

... agreements [that] are renewed or varied many times. [Edgeworth (1925), p. 314.]

Note that the renewal of contracts is consistent with a market that takes place periodically over time. This is also suggested by the fact that Edgeworth chose the labor market to exemplify his concepts. Workers and entrepreneurs show up in the market every period with the same endowment and preferences. In particular, entrepreneurs do not sell the labor units that they bought the previous period. The renewal of contracts is also consistent with the landlord-tenant relationship, another example mentioned by Edgeworth (see below).

Edgeworth is interested in the "final settlements" or what we today call core allocations. A "final settlement" consists of

a set of agreements which cannot be varied with advantage to all the recontracting parties. [Edgeworth (1925), p. 314.]

The market presumably reaches a final settlement after going through various "settlements" or "temporary equilibria." Edgeworth is not clear as to when consumption takes place: whether at each temporary equilibria or only at a final settlement. Indeed, when he explains the meaning of recontracting (or blocking, in the modern economist's jargon) he gives two examples which point to different directions concerning the issue of the timing of consumption $^{9}$ :

Thus an auctioneer having been in contact with the last bidder (to sell at such a price if no higher bid) recontracts with a higher bidder. So a landlord on expiry of lease recontracts, it may be, with a new tenant. [Edgeworth (1881), p. 17.]

\footnotetext{
${ }^{9}$ Emphasis in the original.
} 
In the first case there is no transaction (an a fortiori no consumption) between the auctioneer and the last bidder, due to the appearance of a higher bidder. In the second example, on the other hand, there is a transaction every period between a landlord and a tenant that leads to consumption. Here, although each period's contract is just a settlement, it is not necessarily a final one.

Edgeworth proposed the core as an alternative to the competitive equilibrium allocations, identified several years earlier by Walras (1874). He found that the core was most of the times indeterminate as a solution concept, by prescribing a large set of final settlements; this was contrasted to the more determinate concept due to Walras (believed to be "unique"). Because of this comparison, Edgeworth was especially interested in this question of "uniqueness" of the core, and was the first to notice the connection between the core and Walrasian allocations in large economies. ${ }^{10}$ Thus, for Edgeworth's main purpose, the timing of consumption is irrelevant. One can interpret his temporary equilibria, very much in the Walrasian tattonement tradition, as agreements that are not carried out but which are intermediate steps leading to a final settlement; and one can interpret his settlements as agreements that are carried out each period without affecting the "disposition and circumstances" of the parties for next period. Edgeworth's main point can be understood adopting either interpretation.

However, when one focuses on the dynamic process of recontracting, the interpretation of states of the system is important. The models in Feldman (1974) and Green (1974), since they do not have random perturbations, may potentially (and in fact, actually) converge to an absorbing state. Therefore they are consistent with an interpretation in which consumption only takes place after convergence is achieved. In our model, since agents make random mistakes, the system has no absorbing states. Therefore an interpretation more in line with the landlord-tenant example, in which there are transactions and consumption

\footnotetext{
${ }^{10}$ This observation gave rise to the important core convergence/equivalence literature (Debreu and Scarf (1963), Aumann (1964)) as one of the leading game theoretic justifications of Walrasian equilibrium. See Anderson (1992) and Aumann (1987) for surveys. Although the robustness of the equivalence result is remarkable, several violations thereof have been identified, from which one can learn the role of certain frictions in markets. These references include Anderson and Zame (1997) for infinite dimensional commodity spaces, Manelli (1991) for instances of satiation in preferences, and Serrano, Vohra and Volij (2001) for asymmetric information.
} 
every period, is more appropriate.

Essential to the recontracting process is the idea that any individual is free to contract and recontract without the consent of third parties. But note that within the first interpretation there is no consumption until all agents reach an agreement. That is, no one can consume until we are sure that every agent has reached a final settlement. In fact, it may well be the case that along the path toward the final settlement, some agents hold bundles that are strictly preferred to the bundle that is consumed by them at any final settlement. Postponing consumption until the very end seems to contradict the spirit of recontracting without the consent of others. If one can recontract without others' permission, then it would appear that one should be able to also consume without their consent.

\section{Concluding Remarks}

1. Theorem 1 uses the "global dominance" property of the Walrasian allocation, as specified in Lemma 1, part (iii). Although the models are very different, this dominance of the Walrasian allocation resembles the main driving force of the result in Vega-Redondo (1997). His paper proposes an evolutionary process based on imitation, and its Walrasian result relies on the fact that if a firm produces the competitive output in a symmetric oligopoly, its profit is always higher than that of those firms that produce any other output level.

2. Note that the sufficient condition on the cost of a serious mistake, $\lambda>|N|-2$, used to obtain Theorem 1, is jeopardized when $|N|$ grows. Thus, for a given specification of $\lambda$, the system may get stuck at other allocations because the number of indifferences required to abandon a non-Walrasian allocation to go to the Walrasian allocation grows. If one fixes $\lambda$, making the economy large is an obstacle to the Walrasian result: other allocations could also be visited by the process a positive fraction of time in the long run. This contrasts with the core convergence literature, based on the existence of more blocking coalitions in large economies.

3. Along the same lines, if indifferences are present in the economy, the examples in Section 6 demonstrate that stochastic stability may yield a variety of patterns, and that the long run prediction may be compatible with the presence of market frictions - non-Walrasian allocations. In particular, Example 5 suggest that the core may not be exactly capturing coalitional stability in a model where mistakes are allowed. Thus, dynamic analysis may be 
a useful complement to the set of core allocations, one of the central recommendations of cooperative game theory. It may happen that some regions of the core are hard to access, while some non-core allocations may have strong dynamic attractor properties. ${ }^{11}$

4. Our results are robust if the transition rule in the unperturbed process is that of coalitional weak blocking, instead of the strict blocking specified in $\mathcal{M}^{0}$. As the analysis of the process $\mathcal{M}_{W}^{0}$ in Section 7 shows, one can find conditions under which only some Walrasian allocations are stochastically stable, while in general one must also contemplate the possibility of stochastically stable cycles that include non-Walrasian allocations.

\footnotetext{
${ }^{11}$ In a different setting that includes externalities, Gomes and Jehiel (2005) analyze a dynamic game of coalition formation. In their model, the agents are forward-looking and the Markov perfect equilibria do not provide support to the core. They interpret their result as an instance of how forward-looking agents may disturb the core predictions. In contrast, our agents are myopic, but the stochastic dimension -the presence of mistakes- also leads to questioning the core. See also Ray and Vohra (2001) and Konishi and Ray (2003).
} 


\section{References}

Anderson, R. M. (1992), "The Core in Perfectly Competitive Economies", chapter 14 in R. J. Aumann and S. Hart (eds.) Handbook of Game Theory with Economic Applications (vol. I), North Holland, Amsterdam.

Anderson, R. M. and W. Zame (1997), "Edgeworth's Conjecture with Infinitely Many Commodities: L1", Econometrica 65, 225-273.

Aumann, R. J. (1964), "Markets with a Continuum of Traders", Econometrica 32, 39-50.

Aumann, R. J. (1987), "Game Theory", in J. Eatwell, M. Milgate and P. Newman (eds.) The New Palgrave Dictionary of Economics, Norton, New York.

Ben-Shoham, A., R. Serrano and O. Volij (2004), "The Evolution of Exchange," Journal of Economic Theory 114, 310-328.

Bergin, J. and B. Lipman (1996), "Evolution with State Dependent Mutations," Econometrica 64, 943-956.

Binmore, K. G. (1985), "Bargaining and Coalitions," in Game-Theoretic Models of Bargaining, Roth, Alvin E., ed., Cambridge University Press, New York and Sydney; 269-304.

Dagan, N., R. Serrano and O. Volij (2000), "Bargaining, Coalitions and Competition," Economic Theory 15, 279-296.

Debreu, G. and H. Scarf (1963), "A Limit Theorem on the Core of an Economy", International Economic Review 4, 235-246.

Edgeworth, F. Y. (1881), Mathematical Psychics, Kegan Paul Publishers, London, reprinted in 2003, P. Newman (ed.) F. Y. Edgeworth's Mathematical Psychics and Further Papers on Political Economy, Oxford University Press.

Edgeworth, F. Y. (1925), Papers Relating to Political Economy (Vol. 2), Burt Franklin, New York.

Feldman, A. (1974), "Recontracting Stability," Econometrica 42, 35-44. 
Foster, D. P. and H. P. Young (1990), "Stochastic Evolutionary Game Dynamics," Theoretical Population Biology 38, 219-232.

Freidlin, M. and A. Wentzell (1984), Random Perturbations of Dynamical Systems, Berlin: Springer-Verlag.

Gomes, A. R. and P. Jehiel (2005), "Dynamic Processes of Social and Economic Interactions: On the Persistence of Inefficiencies," Journal of Political Economy 113, 626667.

Green, J. (1974), "The Stability of Edgeworth's Recontracting Process," Econometrica 42, $21-34$.

Kandori, M., G. Mailath, and R. Rob (1993), "Learning, Mutations and Long Run Equilibria in Games," Econometrica 61, 29-56.

Kandori, M., R. Serrano and O. Volij (2004), "Decentralized Trade, Random Utility and the Evolution of Social Welfare," Working Paper 2004-06, Department of Economics, Brown University.

Konishi, H. and D. Ray (2003), "Coalition Formation as a Dynamic Process," Journal of Economic Theory 110, 1-41.

Manelli, A. (1991), "Monotonic Preferences and Core Equivalence", Econometrica 59, 123-138.

Perry, M. and P. J. Reny (1994), "A Non-Cooperative View of Coalition Formation and the Core," Econometrica 62, 795-817.

Ray, D. and R. Vohra (2001), "Coalitional Power and Public Goods," Journal of Political Economy 109, 1355-1384.

Roth, A. and A. Postlewaite (1977), "Weak versus Strong Domination in a Market for Indivisible Goods," Journal of Mathematical Economics 4, 131-137.

Roth, A., T. Sonmez and M. U. Unver (2004), "Kidney Exchange," Quarterly Journal of Economics 119, 457-488. 
Samuelson, L. (1997), Evolutionary games and equilibrium selection, MIT Press, Cambridge, Massachusetts.

Serrano, R., R. Vohra and O. Volij (2001), "On the Failure of Core Convergence in Economies with Asymmetric Information," Econometrica 69, 1685-1696.

Shapley, L. and H. Scarf (1974), "On Cores and Indivisibility," Journal of Mathematical Economics 1, 23-28.

Vega-Redondo, F. (1996), Evolution, games, and economic behavior, Oxford University Press, Oxford, New York.

Vega-Redondo, F. (1997), "The Evolution of Walrasian Behavior," Econometrica 65, 375384.

Walras, L. (1874), Elements of Pure Economics, or the Theory of Social Wealth, English edition (ed. W. Jaffé), Reprinted in 1984 by Orion Editions, Philadelphia.

Weibull, J. (1995), Evolutionary Game Theory, MIT Press, Cambridge, Massachusetts.

Young, H. P. (1993), "The Evolution of Conventions," Econometrica 61, 57-84.

Young, H. P. (1998), Individual Strategy and Social Structure: an Evolutionary Theory of Institutions, Princeton university Press, Princeton, NJ. 